\title{
Neural correlates mediating the consolidation of visuomotor adapted actions: A study on the role of night sleep versus the passage of daytime
}

Debas, Karen ${ }^{1}$; Carrier, Julie ${ }^{2,3}$; Orban, Pierre ${ }^{1}$; Barakat, Marc ${ }^{1}$; Vandewalle, Gilles ${ }^{1}$; Abdallah Hadj Tahar ${ }^{1}$; Karni, Avi ${ }^{4}$; Ungerleider, Leslie G. ${ }^{5}$; Benali, Habib ${ }^{6}$ and Doyon, Julien ${ }^{1,3,5,6}$

${ }^{1}$ Functional Neuroimaging Unit, University of Montreal Geriatric Institute, Montreal, Quebec, Canada

${ }^{2}$ Centre d'étude du sommeil et des rythmes biologiques, Hôpital du Sacré-Cœur de Montréal, Montreal, Quebec, Canada

${ }^{3}$ Centre de recherche en neuropsychologie et en cognition, Department of Psychology, University of Montreal, Montreal, Quebec, Canada

${ }^{4}$ Laboratory for Functional Brain Imaging and Learning Research, The Brain-Behavior Center, University of Haifa, Haifa, Israel

${ }^{5}$ Laboratory of Brain and Cognition, NIMH, NIH, Bethesda, MD, USA

${ }^{6}$ Unité Mixte de Recherche-S 678, Institut National de la Santé et de la Recherche

Médicale/University of Paris 6, Centre Hospitalier Universitaire Pitié-Salpêtrière, Paris,

France

Introduction

Motor skill consolidation refers to the brain plasticity taking place whereby learning results in an enduring change in neural representation. Our group and others have previously demonstrated that sleep contributes to the consolidation of newly acquired motor sequences, but its contribution in consolidating visuomotor adapted movements (MA) remains unclear. Some have reported that the improvement in performance (measured through delayed gains or a faster rate of relearning [i.e., savings]) is sleepdependent (Huber et al., 2004), while others have found time to be sufficient (Doyon et al., submitted; Krakauer et al., 2005). Furthermore, the neural correlates subserving MA consolidation following sleep or the passage of time is unknown.

Methods

To address these issues, we used an eight-target tracking task, for which subjects used a joystick with their dominant hand in order to move a cursor positioned at the center of the screen to one of 8 targets following an elliptical trajectory (Fig1). MA was tested using a 'reversed' condition where the relation between movements of the joystick and direction of the cursor were inverted by 180 degrees on each trial. A control task consisting of a 'direct' condition using 4 targets was also administered. Twenty-four subjects were assigned to two groups: Night/sleep and Day/awake. In the Night/sleep group ( $\mathrm{n}=12)$, subjects were asked to : a) train around 9:00 p.m, b) be scanned immediately after training while performing the task or a rest condition, c) sleep in the lab, and d) be retested while being scanned 12 hours later. In the Day/awake group $(n=11)$, the training and first scanning sessions were carried out at 9:00a.m. Subjects remained awake, and were then retested 12 hours later (Fig2). The control task was administered 1 week apart, in a counterbalanced order, using the same procedure.

$\underline{\text { Results }}$

Behavioural- Both groups improved during training until reaching asymptotic performance. Also, both groups showed savings at retest (Fig3). Univariate analyses revealed a $10 \%$ improvement in precision $(\mathrm{p}=.01)$ and $13 \%$ improvement in precision 
relative to time $(\mathrm{p}=.02)$. Importantly, there were no Group $\mathrm{x}$ Session interaction $(\mathrm{p}>.2)$, suggesting no effect of sleep.

fMRI - Functional images were analysed using SPM2. A conjunction analysis showed that both groups started off with increased activity in M1, striatum and the cerebellum during the immediate post-training session. Effects of consolidation assessed by a conjunction analysis of the two groups in the delayed $>$ immediate contrast yielded activity in the right cerebellar cortex (Lobule VI) (Fig4). A correlation analysis between the latter contrast and the amount of savings revealed activity in an more extended neural network, including Lobule VI in the cerebellum. By contrast, the Group x Session interaction analysis did not reveal any significant activity in motor-related regions.

Conclusion

Our results support the hypothesis that time alone is sufficient to elicit savings in motor adaptation learning and that this sleep-independent process relies on the integrity of Lobule VI in the cerebellum. These findings also support the notion that the cerebellum plays a critical role in the acquisition of an internal model and MA consolidation.

Fig 1. Motor adaptation task: Trajectory in blue is the ideal elliptical curve; in read are a subject's curves

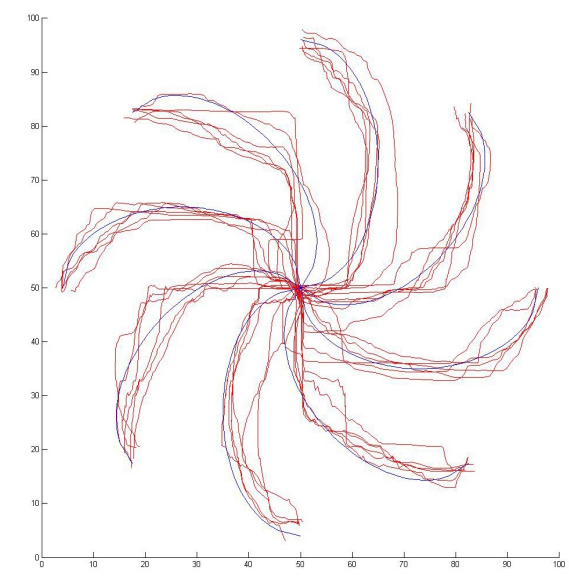

Fig 2. Experimental Design

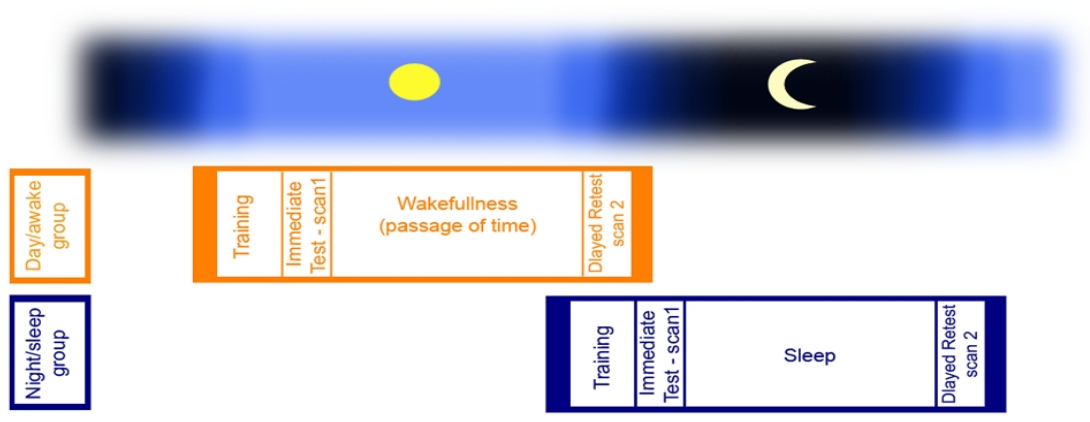


Fig 3. Behavioural results for Night/sleep and Day/awake together

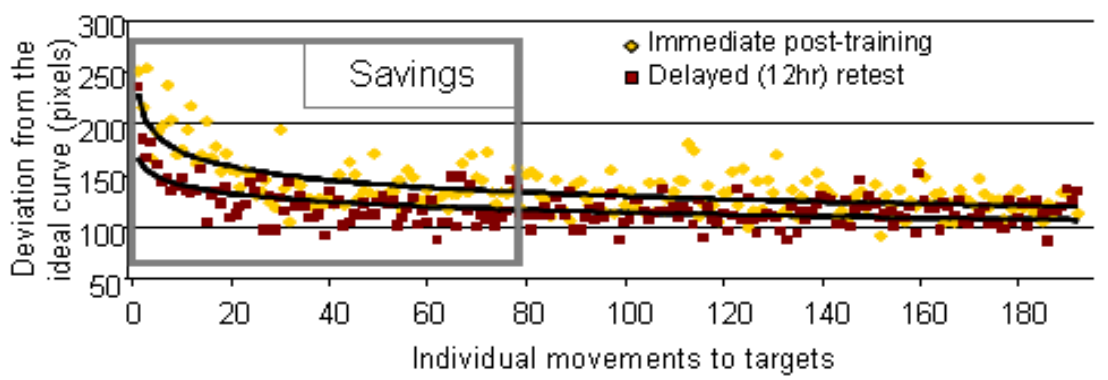

Fig 4. Functional imaging data

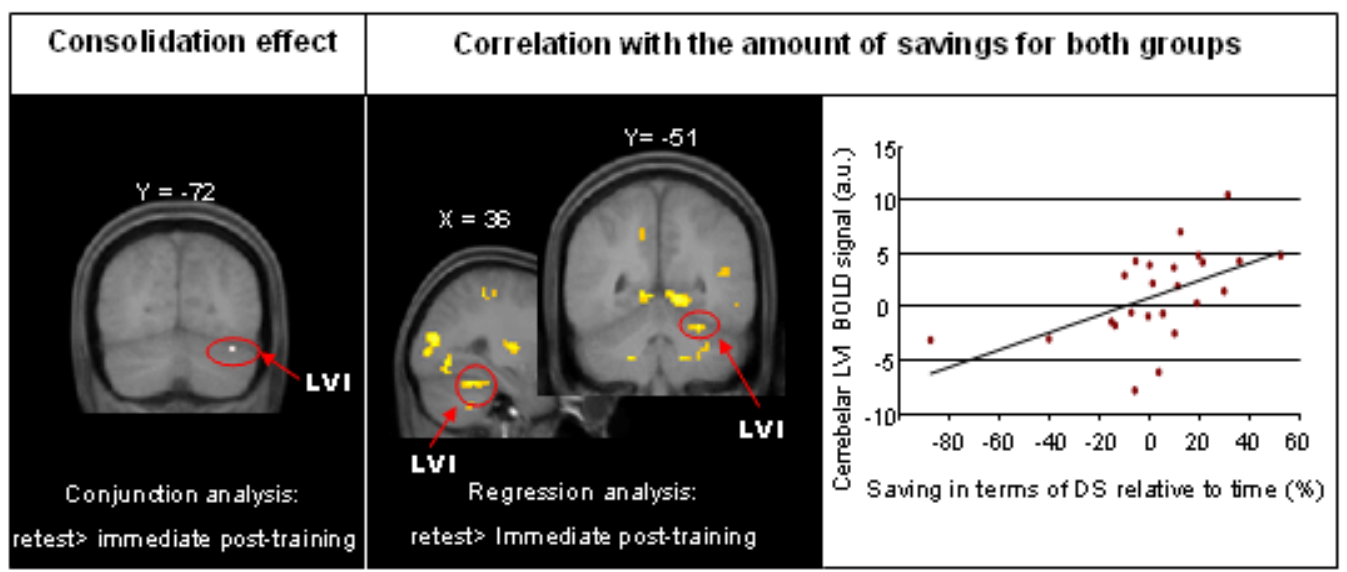

References:

Huber R, Ghilardi MF, Massimini M, Tononi G (2004) Local sleep and learning. Nature 430:78-81.

Imamizu H, Miyauchi S, Tamada T, Sasaki Y, Takino R, Putz B, Yoshioka T, Kawato M (2000) Human cerebellar activity reflecting an acquired internal model of a new tool. Nature 403:192-195.

Krakauer JW, Ghez C, Ghilardi MF (2005) Adaptation to visuomotor transformations: consolidation, interference, and forgetting. J Neurosci 25:473-478. 